\title{
ANÁLISIS
}

\section{APROXIMACIÓN SISTEMÁTICA A LA CREACIÓN DE VERSIONES DIGITALES DE NEGATIVOS FOTOGRÁFICOS HISTÓRICOS}

\section{Systematic approach to the generation of digital versions of historical photographic negatives}

\author{
Jesús Robledano-Arillo y Diego Navarro-Bonilla
}

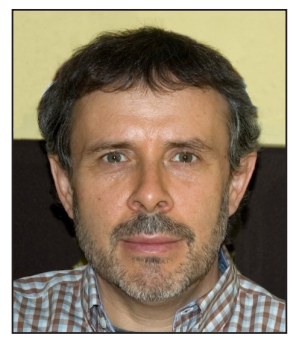

Jesús Robledano-Arillo es docente en la Universidad Carlos III de Madrid desde 1996 en áreas relacionadas principalmente con la documentación audiovisual y tecnologías de la imagen. Doctor en Documentación con premio extraordinario. Director del Máster Universitario en Archivos, Gestión de Documentos y Continuidad Digital. Investigador en 26 proyectos con financiación pública o privada, 18 de ellos como investigador principal. Tiene varios libros y trabajos sobre los campos de su especialidad.

http://orcid.org/0000-0002-4090-8684

jroble@bib.uc3m.es

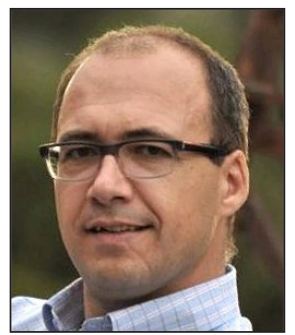

Diego Navarro-Bonilla, doctor en Documentación por la Universidad Carlos III de Madrid (UC3M) y doctor en Conflictos, seguridad y solidaridad por la Universidad de Zaragoza. Profesor titular de Archivística, ex-director del Master en Archivos, Gestión de Documentos y Continuidad Digital y fundador y exdirector del Instituto Juan Velázquez de Velasco de investigación en inteligencia para la seguridad y la defensa (todo ello en la UC3M). Es Premio Nacional de Defensa 2003 y coordinador general del MOOC "Introducción a la caligrafía y paleografía en archivos hispanos medievales y modernos".

http://orcid.org/0000-0002-1782-7202

dnavarro@bib.uc3m.es

Universidad Carlos III de Madrid, Departamento de Biblioteconomía y Documentación C/ Madrid, 126. 28903 Getafe (Madrid), España

\section{Resumen}

Se describe un método para la generación de versiones master de negativos fotográficos históricos que sean altamente representativas de sus originales a partir de capturas realizadas con dispositivos de fácil acceso y costo asumible para cualquier institución cultural. Se aborda la creación de copias positivas virtuales historicistas que puedan ser utilizadas para divulgar su contenido icónico de acuerdo con unos rigurosos criterios técnicos e históricos. La metodología desarrollada ha sido aplicada a una muestra de negativos fotográficos del fondo Skogler (1936-1939). Se han ideado e implementado métodos de corrección de defectos de falta de uniformidad y rango dinámico introducidos por los dispositivos de captura que no acarreen la alteración o pérdida de la información tonal original.

\section{Palabras clave}

Digitalización; Fotografía; Negativos fotográficos; Procesamiento digital de imágenes; Corrección de imágenes digitales; Skogler.

\footnotetext{
Abstract

We describe a method for obtaining master versions of historical photographic negatives that are highly representative at the tonal level of their originals from captures made with easily accessible and affordable devices for any cultural organization. We also address the process of creating historic positive virtual copies that can be used to disseminate iconic content according to rigorous technical and historical criteria. The methodology has been applied to a sample of photographic negatives from the Skogler archive (1936-1939). We devised and implemented corrective processes for defects of non-uniformity or dynamic range introduced by the capture devices that do not result in the alteration or loss of the original tonal information.
} 


\section{Keywords}

Digitization; Photography; Photographic negatives; Digital image processing; Digital image correction; Skogler.

Robledano-Arillo, Jesús; Navarro-Bonilla, Diego (2017). “Aproximación sistemática a la creación de versiones digitales de negativos fotográficos históricos". El profesional de la información, v. 26, n. 6, pp. 1172-1183.

https://doi.org/10.3145/epi.2017.nov.16

\section{Introducción}

La captura digital de negativos fotográficos históricos requiere la obtención de imágenes máster digitales muy representativas con respecto a las características ópticas de los originales. Esta idea se ha reivindicado en los últimos años en la comunidad de expertos en patrimonio fotográfico (Capell, 2010; Mitjà, 2012; Puglia; Reed; Rhodes, 2004; Puglia; Rhodes, 2007; Robledano-Arillo, 2011; 2014; 2017). Las imágenes máster altamente representativas son de gran utilidad para el conocimiento preciso de los originales sin necesidad de manipularlos y para apoyar las tareas de su preservación, al aportar datos sobre su estado físico en el momento de la captura con una alta precisión; pero su captura y codificación son más complejas y costosas en recursos, pericia y tiempo. Otro problema añadido es la inexistencia de unos criterios técnicos y principios firmes y bien asentados en la comunidad del patrimonio cultural que puedan ser tomados como referencia para la codificación digital rigurosa de este tipo de materiales.

El presente trabajo deriva de la necesidad de abordar la digitalización urgente de una fracción del fondo de negativos del estudio fotográfico Skogler ${ }^{1}$ en manos de un coleccionista privado, ante el mal estado de conservación de los originales y la necesidad de estudiar y difundir su contenido.

\section{La captura digital de negativos fotográ- ficos históricos requiere la obtención de imágenes master digitales muy repre- sentativas de las características ópticas de los originales}

Se trata de negativos sobre base plástica al gelatinobromuro de plata en formato $6 \times 9 \mathrm{~cm}$ tomados en plena Guerra Civil durante el período 1936-1938 y que muestran escenas y lugares de la zona de retaguardia en la ciudad de Zaragoza y alrededores. Nuestro primer objetivo ha sido desarrollar y validar una metodología para un flujo de trabajo de codificación de los masters que, con dispositivos de captura de fácil acceso y bajo coste, garantice una alta precisión en la representación digital de las densidades ópticas de los originales. El segundo objetivo ha sido idear una metodología para la generación de versiones positivas virtuales historicistas a partir de los masters ${ }^{2}$. Aunque se da solución a las necesidades de un proyecto concreto, este planteamiento metodológico y técnico será de utilidad a otras instituciones que tengan que enfrentarse a trabajos similares.

Hemos excluido el tratamiento del color dentro de los objetivos del proyecto, pese a su relevancia como característica física esencial de los negativos históricos. El motivo ha sido el gran desarrollo requerido para tratar los aspectos anteriores.

\section{Estado de la cuestión}

Desde finales de la década de 1990 existen estudios que defienden la captura digital de materiales fotográficos con valor patrimonial con las máximas garantías de fidelidad hacia los originales y de calidad.

Reilly y Frey (1996) y Frey y Reilly $(1999 ; 2006)$ fueron pioneros sistematizando el tipo de información intrínseca de una imagen fotográfica que hay que considerar y evaluar en la captura digital de este tipo de materiales, identificando cuatro tipos de atributos:
- tonales (niveles de densidad óptica);
- color;
- información a nivel espacial (detalle); y otros
- aspectos y problemas de imagen.

En 2004 la National Archives and Records Administration (NARA) publicó las directrices técnicas de digitalización de materiales de archivo (Puglia; Reed; Rhodes, 2004), que describen los requisitos técnicos de captura y codificación de negativos fotográficos y discuten las características de un fichero máster de preservación frente a otro de producción, teniendo el primero unos altos requisitos de representatividad de los originales. En la digitalización de negativos históricos es preciso hacer masters de preservación, debido a la dificultad de mantener las características tonales y densitométricas de los originales a lo largo del tiempo, dada su extrema fragilidad. Esta necesidad justifica el mayor consumo de recursos en la captura, almacenamiento y procesamiento digital que son requeridos para este tipo de masters (Robledano-Arillo, 2016)

Existe también un amplio grupo de estándares y especificaciones enfocados al control de calidad de las imágenes digitales patrimoniales de documentos y obras de arte y sus equipamientos de captura (ISO, 2017a, 2017b; Van-Dormolen, 2012; Nationaal Archief, 2010; Fadgi, 2010; 2016). A pesar de su gran utilidad para evaluar y desechar dispositivos de captura, no aportan métodos para la corrección de defectos que impiden la representatividad precisa de los originales.

Una institución cultural no tiene siempre a su alcance dispositivos con un alto rendimiento en calidad, por lo que se requieren métodos de corrección que permitan acercar los registros digitales con distorsiones reversibles a las características ópticas de los originales. Esta laguna ha sido objeto de investigación en las dos últimas décadas, con estudios que proponen métodos de corrección utilizables en ámbitos 
profesionales con gran exigencia de fidelidad. Aunque no todos estos trabajos provienen del entorno del patrimonio cultural, sus aportaciones tienen plena aplicación en este contexto.

\section{El procesado HDR es costoso en tiempo, pero permite obtener imágenes master con toda la información de densidades de los negativos más contrastados}

Una línea interesante se ha centrado en la corrección digital de negativos fotográficos deteriorados (Landon; Duncan; Seales, 2009; Landon, 2015). Otra en la corrección de distorsiones geométricas de los documentos durante la captura digital (Zhang; Zhang; Tan, 2008; Brown; Seales, 2001; 2004; Cao; Xiaoqing; Liu, 2003). Hay asimismo una amplia línea de investigación centrada en dos graves y frecuentes problemas que impiden un registro correcto de los valores de densidad de los originales: la falta de uniformidad (inhomogeneidad) de tipo espacial y temporal de los dispositivos de captura y sus sistemas de iluminación. Tradicionalmente se ha venido usando un método muy preciso de corrección de inhomogeneidad espacial que toma como referencia una imagen de calibración, consistente en la imagen digital de un fondo blanco uniforme iluminado de la misma manera que lo serán los originales a capturar. A partir del análisis automatizado de esta imagen de referencia, que representa fielmente el patrón de caída de luz a nivel espacial, se construye una LUT (look up table) que contiene los factores de corrección de cada píxel de la imagen. Este cálculo se hace midiendo la diferencia píxel a píxel entre el máximo valor que puede tomar el píxel y el valor que toma en la imagen de referencia. Estos métodos pueden aproximarse mediante las ecuaciones 1 y 2 (Yu, 2004).

$$
\operatorname{LUT}(i, j)=\frac{I r \_ \text {max }}{\operatorname{Ir}(i, j)}
$$

$\forall j=0,1, \ldots m-1 ; i=0,1, \ldots n-1$

$I^{\prime}(i, j)=I(i, j) \operatorname{LUT}(i, j)$

$\forall j=0,1, \ldots m-1 ; i=0,1, \ldots n-1$

\section{Donde}

$\operatorname{LUT}(i, j)=$ factor de corrección a nivel de píxel a almacenar en una LUT;

$\operatorname{lr}(i, j)=$ valor de intensidad en la posición de píxel $(i, j)$ de la imagen de referencia;

Ir_max = valor máximo de $\operatorname{Ir}(i, j)$;

$m=$ alto en pixels,

$n=$ ancho en pixels;

$I(i, j)$ e $I^{\prime}(i, j)=$ valores de intensidad en la posición de píxel $(i, j)$ antes y después de la corrección.

Este tipo de procesados es altamente preciso, al trabajar sobre datos reales a nivel de píxel, pero cuando se aplica a imágenes de gran resolución se vuelve ineficiente en términos de tiempo de proceso. En la bibliografía especializada podemos encontrar otros algoritmos de corrección espacial más complejos e intensivos en tiempo que también parten del análisis de imágenes de calibración previas, como la toma de dos campos de iluminación uniforme, claro y oscuro (flat field correction) (Arjona et al., 2004; Schoonees; Palmer, 2009).

Existen abundantes trabajos de métodos de corrección de falta de uniformidad espacial en el área del procesamiento digital de imágenes médicas de microscopía, resonancias magnéticas e imagen infrarroja, que tratan de evitar la intensidad de procesado de los métodos tipo LUT (Tomaževič; Likar; Pernuš, 2002; Leong; Brady; McGee, 2003; Likar et al., 2000, Likar; Viergever; Pernuš, 2001; Yu, 2004; Hardie et al., 2009).

En el área de la imagen infrarroja en movimiento hay una productiva vía de trabajo que trata de resolver la corrección de inhomogeneidades temporales en secuencias en tiempo real y cuyas aportaciones podemos aplicar a la digitalización en serie de documentos. Estos métodos, comúnmente denominados scened based nonuniformity correction, no utilizan imágenes de referencia sino el análisis estadístico de la respuesta de cada píxel en escenas reales capturadas por la cámara en pleno funcionamiento (Hayat et al., 1999; Huang et al., 2015).

En el presente trabajo no hemos considerado necesario acudir a métodos del tipo scened based porque al poder trabajar con unas condiciones de captura controladas donde el factor tiempo no es relevante, hemos podido implementar y aplicar una técnica basada en el uso de una imagen de referencia.

\section{Metodología y resultados}

\subsection{Selección, captura y análisis densitométrico de una muestra representativa}

Un primer paso ha sido analizar la densidad óptica de los negativos. La densidad óptica será expresada a lo largo de este trabajo a través de unidades od (optical density), de acuerdo con la ecuación 3.

$$
o d=-1 \log _{10} \frac{I}{I}
$$

Siendo $I$ ' el flujo luminoso que transmite o refleja una superficie, e I el flujo luminoso incidente.

Este conocimiento se requiere para evaluar la capacidad del sistema de captura para poder registrar correctamente todos los valores de densidad de los originales. Para caracterizar densitométricamente los originales hemos analizado con un densitómetro digital una muestra representativa de 12 originales, concluyendo que podemos tener originales con márgenes de densidad en torno a 2,4 od.

Hemos digitalizado esta muestra además de varias cartas de control tonal con la misma configuración y valores de captura y registro digital que los originales: Stouffer T 3110 calibrada, Stouffer TP 35 calibrada, Kodak IT8 y Danés Picta FS1. La finalidad de estas capturas ha sido el control de 


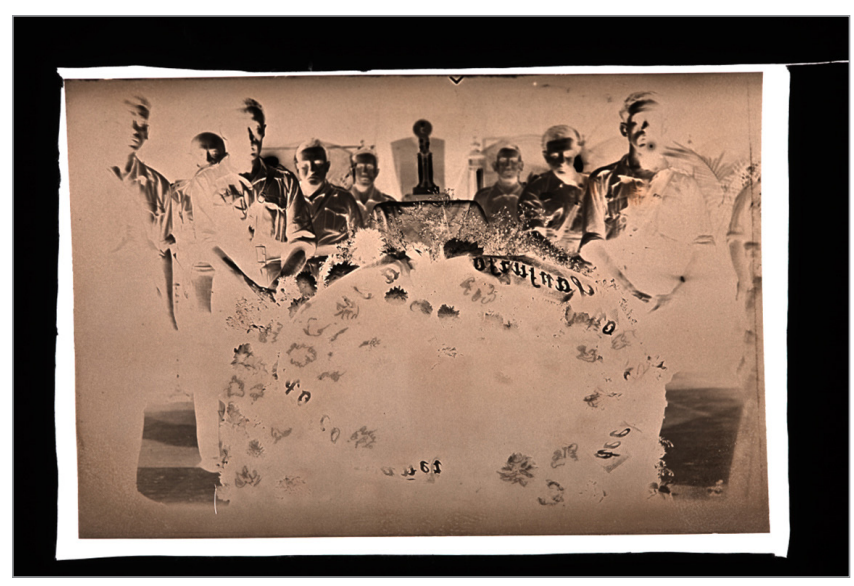

Figura 1. Disposición de captura

calidad del dispositivo de captura y del procesado aplicado a las imágenes, así como la validación de los algoritmos de corrección que describimos más abajo.

Las muestras se han capturado obteniendo directamente de la cámara digital un formato de fichero raw, procesado con el programa DCRaw. Los parámetros de procesado raw han sido: - $v$-o 0 -4 -w -T para obtener una imagen final lineal en formato TIFF no renderizada a 16 bits por canal, sin más procesos que el demosaicing y un balance de blancos previo basado en el balance de blancos de cámara sobre una imagen de una carta de grises. La resolución espacial de muestreo ha sido de 982 pixels por pulgada, la más amplia obtenible con el dispositivo en la configuración usada para la captura.

Las capturas se han realizado con una mesa de luz de alta calidad (Just Normlicht-Color Control Classic Line DI 1 Evg). Hemos usado una cámara digital Canon EOS $5 d$ con sensor de $4.368 \times 2.912$ pixels (DS1260091 DC 8.1V, n. serie $2631201434)$ y equipada con una óptica Canon EF100mm f/2.8 Macro USM, montada en un stand de reproducción. Para el mejor posicionamiento de los originales en la mesa de luz se ha utilizado una plantilla de cartulina de color negro con la forma y tamaño de los negativos. En la figura 1 mostramos la disposición de captura con un negativo concreto.

\subsection{Control de distorsiones que afec- tan a la calidad tonal}

El siguiente paso ha sido caracterizar el rendimiento del dispositivo y controlar sus desviaciones a nivel tonal. Este conocimiento es imprescindible para abordar el desarrollo de los algoritmos de corrección.

\subsubsection{Linealidad}

Este parámetro de calidad permite conocer si la respuesta del dispositivo de captura es perfectamente proporcional a los valores de intensidad de luz. La precisión de la representación de las densidades ópticas del original en la imagen digital depende de que el dispositivo tenga una respuesta lineal, o en su defecto, requerimos el conocimiento de sus desviaciones para poderlas corregir o tenerlas en cuenta en los algoritmos de corrección. Para su medida se han usado las imágenes de la carta TP35. En la figura 2 se presenta la comparativa entre el valor teórico digital y el valor real del parche. Se han normalizado los datos mediante las ecuaciones 4 y 5 . La normalización trata de producir un gráfico lineal del valor de gris normalizado como una función de la densidad óptica, de manera que se pueda apreciar con claridad la desviación de los valores digitales de los parches de densidad con respecto a sus valores teóricos. Los datos son el promedio de los canales RGB para la superficie de cada parche. En el eje de las abscisas se presenta el rango de valores de od de cada parche, en el de las ordenadas los valores correspondientes tras su normalización.

$$
v t d^{\prime}=-1 \log _{10}\left(\frac{\left(2^{16}-1\right) / 10^{o d}}{2^{16}-1}\right)
$$

Donde

od = densidad óptica

$v t d^{\prime}=$ valor teórico digital normalizado

$$
v d^{\prime}=-1 \log _{10}\left(\frac{v d}{2^{16}-1}\right)
$$

\section{Donde}

od = densidad óptica

$v d^{\prime}=$ valor digital normalizado

$v d=$ valor digital no normalizado

Un paralelismo de las líneas roja y azul del gráfico de la figura 2 es significativo de una buena linealidad. La linealidad para los 3 canales RGB se puede considerar buena. Aunque desde el valor de densidad 2 od la respuesta es irregular y menos precisa, esta afectación incide sobre las densidades menos representadas en los originales.

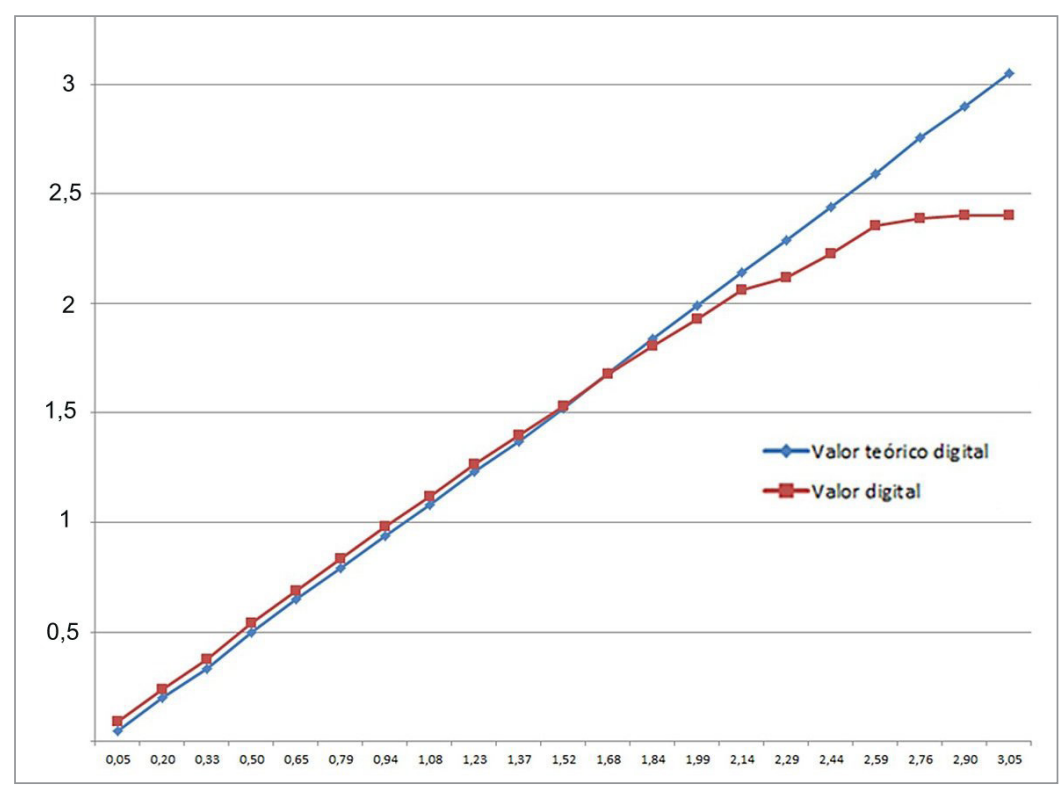

Figura 2. Gráfico lineal del valor del píxel como una función de la densidad óptica 


\subsubsection{Rango dinámico y ruido}

Para la medida del rango dinámico se ha usado la carta TP35. El rango dinámico representa la amplitud del rango de intensidad de luz registrable correctamente en un dispositivo de captura. Se ha tomado como límite de densidad máxima el parche anterior a la zona de recorte, considerando ésta los parches que presentan un valor idéntico de píxel pese a tener densidades ópticas diferentes. También hemos descontado del rango dinámico los niveles de densidad cuyos parches presentan en la imagen digital un elevado nivel de ruido tras aplicar una corrección gamma típica de 2,2. Denominamos ruido a las desviaciones aleatorias del valor de píxel que distorsionan la señal original. Se ha medido el ruido en f-stops, de acuerdo con la ecuación 6 , usando el programa Imatest ${ }^{\circledR}$ para su obtención. Hemos descartado valores de ruido superiores a 0,1 .

$$
r=\frac{\sigma}{\Delta p / \Delta d}
$$

\section{Donde}

$\sigma$ representa la desviación estándar del conjunto de valores de píxel del parche de densidad

$\Delta p$ la diferencia de nivel de pixel del parche de densidad a medir con respecto al anterior

$\Delta \mathrm{d}$ el cociente entre la densidad óptica del parche y el equivalente en densidad óptica de un f-stop $(0,301$ od)

El rango dinámico así medido abarca, con una alta calidad en lo que respecta a la relación señal/ruido, 6,25 f-stops, o lo que es lo mismo 1,9 od. Esta prueba nos lleva irremediablemente a concluir que precisamos una captura HDR (high dynamic range) para poder registrar con calidad toda la gama tonal de muchos de los negativos presentes en el fondo, que como se apunta más arriba, puede llegar a 2,4 $o d$, pues no podemos hacer frente al uso de dispositivos que capturen con el rango dinámico requerido para éstos.

El procesado HDR es costoso en tiempo, pero permite obtener imágenes master con toda la información de densidades de los negativos más contrastados bien representada cuando no se dispone de un equipamiento de alta calidad en rendimiento tonal. Para obtener las imágenes HDR se han realizado cinco tomas: toma estándar ( $f 5,6$ y 1/30), +1 valor de exposición (VE) y +2 VE para las zonas de sombra; y -1 VE y -2 VE para las zonas de luces. Se ha obtenido una imagen HDR de cada negativo a 32 bits por canal. A continuación se ha mapeado cada imagen HDR a 16 bits por canal mediante el método de exposición y gamma, empleando Adobe Photoshop CS3, ajustado como exposición 0 y gamma 1,80. El ajuste se ha hecho tomando como referencia la imagen lineal a exposición 1/30 f/5,6, de manera que se hagan corresponder en luminosidad los valores correspondientes a las mismas zonas en la salida a 16 bits. Para buscar la correspondencia se han seleccionado muestras de superficies de altas luces, medios tonos y sombras.

\subsection{Caracterización espacial y temporal de la unifor- midad}

Los dispositivos de captura suelen presentar con mayor o menor incidencia dos defectos graves de uniformidad que es preciso detectar y subsanar cuando se pretende generar imágenes digitales máster fielmente representativas a nivel óptico de la vista captada del original fotográfico: la inhomogeneidad espacial y la temporal.

La primera alude a que la intensidad de luz no es homogénea en toda el área de captura, de manera que una teórica superficie con un tono homogéneo perfecto quedaría representada en la imagen digital con diferentes valores tonales. La segunda se refiere a variaciones temporales aleatorias en la intensidad de iluminación.

Para medir ambas inhomogeneidades hemos procedido a realizar 16 tomas consecutivas de la mesa de luz, con la misma intensidad de iluminación y exactamente con los mismos ajustes de captura, una detrás de otra inmediatamente, no mediando un lapso de más de 4 segundos entre toma y toma y sin variar en nada las condiciones de toma usadas en las fotografías de la muestra y las cartas de control. Posteriormente hemos procedido a realizar un mapa de áreas de intensidad de luz homogéneas en un rango de 1 a 5 niveles de intensidad de luz sobre las 16 imágenes. Para este proceso hemos usado la aplicación Imatest $^{\circledR}$

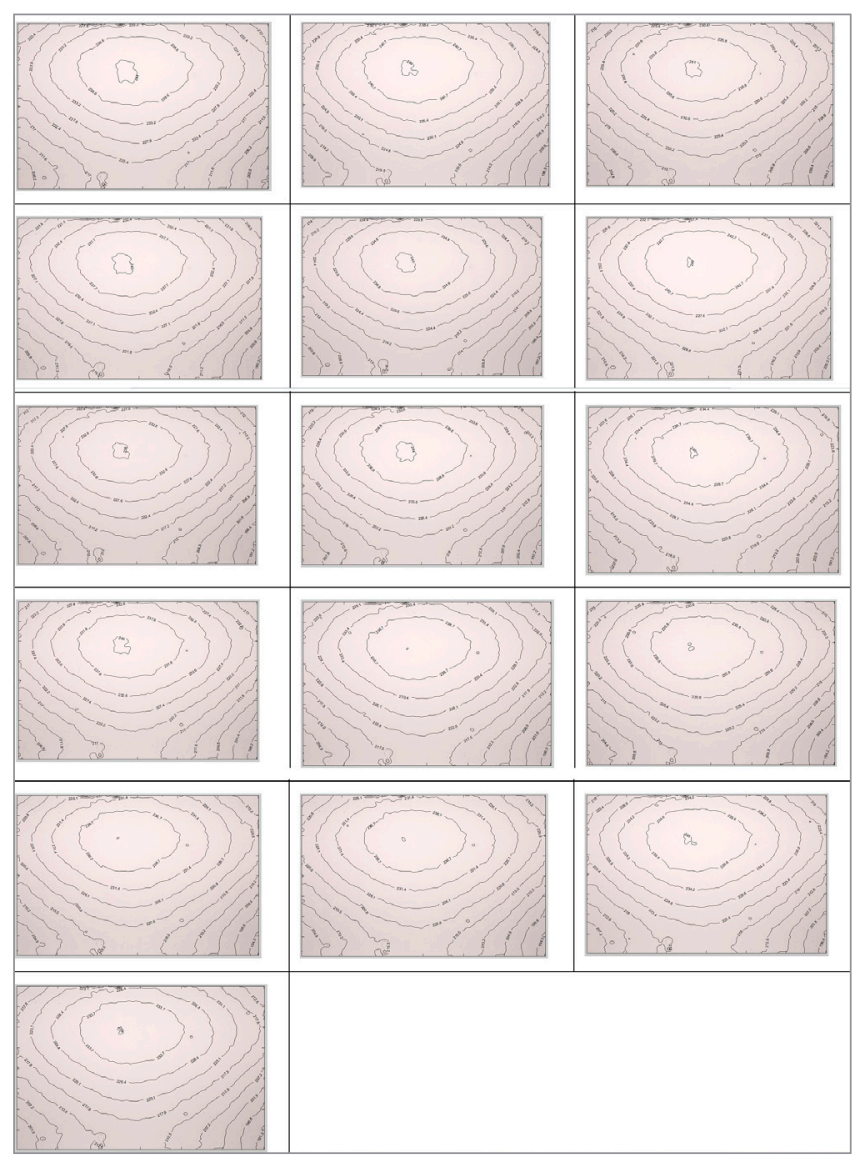

Figura 3. Mapas de zonas de luz homogéneas de las 16 imágenes consecutivas de la prueba de uniformidad espacial y temporal (imágenes 0956 a 971) 


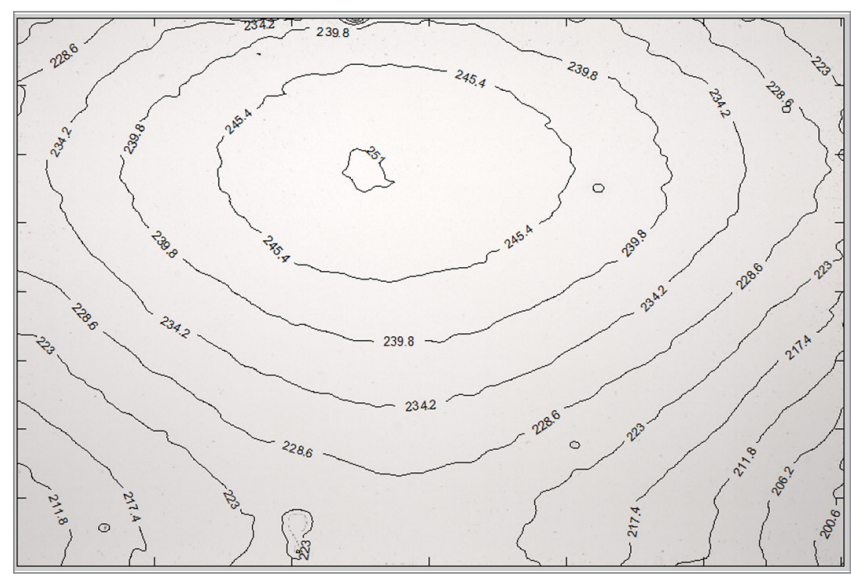

Figura 4. Mapas de zonas de luz homogéneas de una imagen de la mesa de luz resultado de un procesado HDR

Master, en su módulo Light Falloff. El mapa de áreas de la misma intensidad nos va a evidenciar dos cosas: el patrón de caída de luz y si éste es homogéneo toma a toma. En la figura 3 se han incluido los mapas de las 16 tomas consecutivas.

También hemos hecho una imagen HDR (exactamente con el mismo método usado para las fotografías de la colección a capturar) de la mesa de luz, para comprobar si el algoritmo de procesado HDR y de obtención de la imagen LDR permite mantener el mismo patrón de uniformidad de las capturas no HDR. El resultado, mostrado en la figura 4, evidencia que en las imágenes provenientes de procesado HDR el patrón de inhomogeneidad es prácticamente idéntico al del procesado no HDR.

Las imágenes de las figuras 3 y 4 evidencian ambos tipos de inhomogeneidades, siendo especialmente graves y con una alta capacidad de distorsionar la calidad de cualquier proceso de caracterización tonal y cromática del sistema de captura. La inhomogeneidad es provocada por diversos factores: el rendimiento de las lentes, del propio sensor y de la mesa de luz o sistema de iluminación. De acuerdo con los gráficos, la caída de iluminación es casi simétrica en las cuatro esquinas, por lo que hemos de asumir como causa principal el viñeteado provocado por el objetivo utilizado. Estamos ante un serio problema que hay que corregir en las imágenes capturadas si se desea conseguir fidelidad e imágenes representativas a nivel físico o se van a utilizar las cartas de control para realizar correcciones, control de calidad o conseguir datos sobre representación física de los originales. Hemos comprobado también que el patrón de bajada/aumento de luminosidad en las 16 imágenes es perfectamente proporcional y lineal, manteniendo siempre la misma pauta de variación espacial. Gracias a esto se pue- de habilitar un algoritmo más sencillo que suba o baje la luminosidad proporcionalmente para todos los pixels de la imagen en función de la bajada o subida con respecto a una imagen de referencia de valor de intensidad de iluminación conocido.

El siguiente paso ha sido analizar si la temperatura de color se mantiene estable en todas las zonas de caída de luz. Se ha tomado la imagen más luminosa de la serie de 16 y convertido la imagen a 8 bits por canal para la simplificación de los cálculos. Hemos tomado 20 muestras de 189×108 pixels por cada zona de caída de luz del mapa generado previamente. Con este tamaño de muestra se minimiza el efecto del ruido. Posteriormente se ha calculado, muestra a muestra, el promedio de valor de píxel por cada canal RGB y la diferencia entre los valores promedio del canal rojo con respecto al verde y al azul ( $R-G$ y $R-B$ ). Seguidamente hemos calculado la desviación estándar del conjunto de valores de las diferencias, dando como resultado para R-G 1,02 y para $\mathrm{R}-\mathrm{B} 0,88$. Podemos considerar que la temperatura de color se mantiene con suficiente precisión en las diferentes zonas espaciales, por lo que podemos concluir que ésta es homogénea a nivel espacial.

\section{Los dispositivos de captura suelen pre- sentar defectos graves de uniformidad que es preciso detectar y subsanar}

También se ha calculado si la temperatura de color se mantiene estable en el tiempo. Para ello se han tomado las 16 imágenes de la serie temporal, y registrado el valor de dos pixels concretos imagen a imagen, coincidiendo las coordenadas de cada uno en cada una de las imágenes. Hemos hallado la diferencia entre los valores rojo con respecto al verde y rojo con respecto al azul (R-G y R-B) por cada uno de los pixels analizados y para todas las imágenes de la serie. Seguidamente se ha calculado el promedio y la desviación estándar del conjunto de valores de las diferencias R-G y R-B para todas las imágenes de la serie temporal. También se ha calculado la desviación estándar por cada canal RGB en una zona de varios pixels vecinos de 30 pixels alrededor de cada uno de los pixels analizados en todas las imágenes de la serie, y promediado los valores obtenidos. Se incluyen los resultados en la tabla 1.

De acuerdo con los datos de la tabla 1, la temperatura de color se mantiene con suficiente precisión en el tiempo para las diferentes zonas, pues los valores de variación se aproximan a la desviación estándar del valor de cada canal alrededor de cada uno de los pixels. Esta característica nos permitirá sim-

Tabla 1. Datos de análisis de variación temporal de la temperatura de color en la imagen de la mesa de luz tomada en 16 tiempos diferentes

\begin{tabular}{|c|c|c|c|c|c|c|c|}
\hline Píxel & $\mu($ Dif.R-G) & $\boldsymbol{\mu}$ (Dif. R-B) & $\sigma($ Dif. R-G) & $\sigma($ Dif. R-B) & $\mu(\sigma($ Canal R)) & $\mu(\sigma($ Canal G) $)$ & $\mu(\sigma($ Canal B $))$ \\
\hline 1 & 10,75 & 12,56 & 1,52 & 1,62 & 1,62 & 1,55 & 1,6 \\
\hline 2 & 10,81 & 12,13 & 1,84 & 1,87 & 1,31 & 1,13 & 1,32 \\
\hline
\end{tabular}


plificar los algoritmos de corrección, al evitarnos tener que ponderar incluir procesados adicionales para reajustar los datos de imagen corregidos en uniformidad a la temperatura de color original del píxel antes de la corrección.

\subsection{Corrección de uniformidad temporal}

Como las variaciones en intensidad de luz diacrónicas son perfectamente lineales manteniendo en el tiempo el mismo patrón de inhomogeneidad espacial, la computación de la igualación temporal se hace relativamente sencilla a través de un procesado que emplea una imagen de referencia (imagen patrón), coincidente con la captura más luminosa de la mesa de luz, y zonas de referencia extraídas de ésta y de cada una de las imágenes capturadas (grupo patrón). En la figura 5 se muestra el grupo de pixels patrón, que conforma un área rectangular cuyas coordenadas representan en todas las imágenes digitalizadas una porción de la mesa de luz completamente libre de cualquier elemento superpuesto.

La ecuación 7 se corresponde con el algoritmo de corrección temporal. El procedimiento se basa en hacer el promedio de los pixels del grupo patrón en la imagen patrón y en todas las imágenes capturadas por cada canal RGB. Hallando la diferencia de valor entre el promedio del grupo patrón de la imagen patrón y el promedio del resto de las imágenes, por cada canal, se obtienen tres valores numéricos redondeados que representan la diferencia temporal de todas las imágenes con respecto a la imagen patrón medida por cada canal RGB. Esos tres valores se ponderan posteriormente para modelar el efecto de la reducción de intensidad de luz proporcional a la densidad óptica de la superficie de la fotografía original que corresponde al píxel. Con esos valores numéricos ponderados se escalan los valores de cada canal RGB de todos los pixels de las imágenes correspondientes a las capturas de las fotografías del fondo.

$$
I^{\prime}(j, i, k)=I(j, i, k)+\operatorname{round}\left(f t(k)\left(\frac{I q(j, i, k)}{P(j, i, k)-f t(k)}\right)\right)
$$

$\forall j=0,1, \ldots m-1 ; i=0,1, \ldots n-1 ; k=1,2,3$

$\forall>300$

Donde

$f t(k)=$ factor de corrección temporal para el canal $k$,

$I$ = imagen a corregir temporalmente,

$I q=$ imagen a corregir temporalmente no corregida espacialmente,

$P=$ imagen patrón corregida en ruido y suciedad pero no espacialmente,

$I^{\prime}=$ Imagen corregida temporalmente,

$m=$ alto en pixels,

$n=$ ancho en pixels $y$

$k=$ canal

Donde

$$
f t(k)=\frac{1}{m n} \sum_{k=1}^{k} \sum_{m=0}^{m-1} \sum_{n=0}^{n-1} P p(j, i, k)-G p(j, i, k)
$$

$\forall j=0,1, \ldots m-1 ; i=0,1, \ldots n-1 ; k=1,2,3$

Donde

$G p=$ grupo patrón en la imagen a corregir,

$P p=$ grupo patrón en la imagen de referencia,

$m=$ alto en pixels del grupo patrón,

$n=$ ancho en pixels del grupo patrón,

$k=$ canal RGB

\subsection{Corrección de uniformidad espacial}

La eficacia de esta corrección aumenta si se realiza tras la corrección temporal. El método de corrección propuesto entra dentro de la categoría de los métodos que más arriba denominamos como basados en LUT. Partimos del método clásico acorde con las ecuaciones 1 y 2 , pero lo hemos ampliado para evitar introducir en la imagen corregida procesamiento tipo ghosting (improntas de elementos de otras imágenes en la imagen a corregir) cuando la imagen de referencia tiene detalles de alto contraste provocados por motas y suciedad presentes en la mesa de captura, y para poder obtener datos precisos que permitan conocer a fondo la falta de uniformidad.

\section{Primer paso. Caracterización espacial de la imagen de re- ferencia}

El procedimiento parte de la detección en la imagen patrón de referencia de la zona más clara y uniforme, promediándose a continuación sus valores de píxel por cada canal RGB. A estos promedios los denominados como MáximoR, MáximoG y MáximoB; y a esta zona como zona patrón o píxel de referencia para la corrección espacial. El que se utilice la zona más clara de la imagen patrón no representa riesgo de saturación de ningún canal de las imágenes de los negativos originales debido a que en éstos la superficie más clara corresponde al velo más el soporte, que es siempre necesariamente más densa que la luz de la mesa de luz. Se hace preciso corregir el área que representa la mesa de luz en la imagen patrón antes del cálculo de los factores de corrección, eliminando la representación digital de la suciedad o exceso de ruido, debido a que la impronta de estos elementos puede llegar a superponerse a las imágenes corregidas. Se ha usado el filtro de eliminación de polvo de Adobe Photoshop CS5 configurado con un radio 14 y un umbral 0.

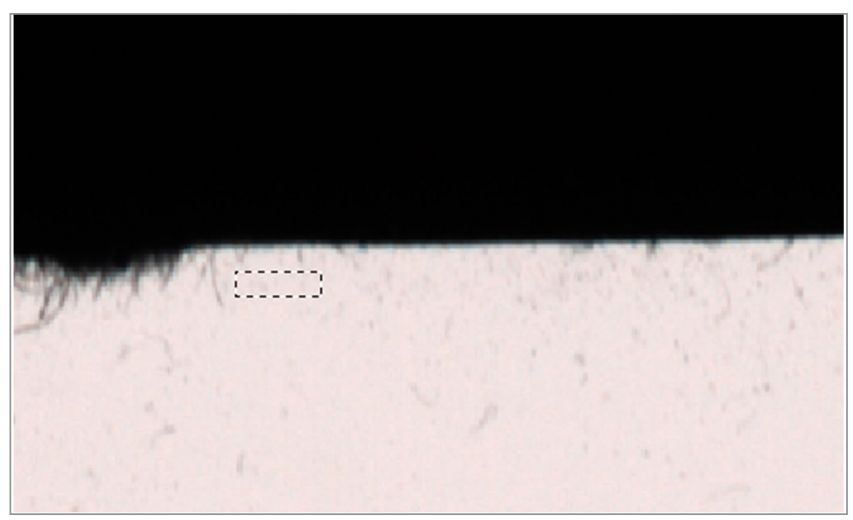

Figura 5. Grupo patrón de la imagen patrón para la estimación del factor de corrección temporal. Detalle. 
Tabla 2. Diferencia de promedios de valores de parche entre parches de densidad similar o casi similar de dos cartas diferentes

\begin{tabular}{|c|c|c|c|c|c|c|}
\hline \multirow{2}{*}{ Número } & \multirow{2}{*}{ Parches T3110 } & \multirow{2}{*}{$\begin{array}{l}\text { Parches TP35 } \\
\text { equivalente }\end{array}$} & \multicolumn{4}{|c|}{ Diferencia de valor promedio de píxel en valores absolutos } \\
\hline & & & ET & No ET & HDR ET & HDR no ET \\
\hline 1 & $1(0,04$ od $)$ & $1(0,05$ od $)$ & 6,49 & 24 & 10,61 & 21 \\
\hline 2 & $7(0,66$ od $)$ & $5(0,65$ od $)$ & 2,06 & 6 & 1,17 & 6 \\
\hline 3 & $10(0,95$ od $)$ & $7(0,94$ od $)$ & 0,64 & 3 & 0,27 & 3 \\
\hline 4 & $13(1,24$ od $)$ & $9(1,23$ od $)$ & 0,3 & 2 & 0,01 & 3 \\
\hline 5 & $16(1,51$ od $)$ & $11(1,52$ od $)$ & 0,14 & 0 & 0,48 & 2 \\
\hline 6 & $19(1,8$ od $)$ & $13(1,87$ od $)$ & 0,09 & 1 & 0,49 & \\
\hline & & Promedio & 1,62 & 6 & 2,17 & 6 \\
\hline & & $\begin{array}{l}\text { Desviación } \\
\text { estándar }\end{array}$ & 2,28 & 8,27 & 3,8 & 6,88 \\
\hline
\end{tabular}

Segundo paso. Cálculo del factor de corrección de cada píxel de la imagen y corrección

El cálculo se basa en restar a MáximoR/G/B el valor de R/G/B correspondiente por cada píxel de la imagen patrón. Pero para que el factor de corrección se adecúe a las imágenes digitales de las fotografías originales se hace preciso ponderarlo, de manera que sea proporcional a la densidad óptica del área de la superficie del original cubierta por el píxel a corregir. El algoritmo de corrección basado en el factor de corrección descrito aparece representado en la ecuación 8.

$$
I^{\prime}(j, i, k)=I(j, i, k)+\operatorname{round}\left((M(k)-P(j, i, k))\left(\frac{I(j, i, k)}{P(j, i, k)}\right)\right)
$$

$\forall j=0,1, \ldots m-1 ; i=0,1, \ldots n-1 ; k=1,2,3$

Donde

I = imagen a corregir espacialmente ya corregida temporalmente,

$I^{\prime}=$ Imagen corregida espacialmente,

$P=$ imagen patrón corregida en ruido y suciedad,

$M=$ Máximo de referencia para cada canal $(k) 1$ (rojo), 2 (verde) y 3 (azul),

$m=$ alto en pixels

$n=$ ancho en pixels

$k=$ canal

\subsection{Validación de las correcciones}

Se han aplicado varios métodos de validación, obteniendo de todos ellos los mismos resultados positivos. Presentamos a continuación uno de ellos, que toma como referencia para el análisis las imágenes de las cartas de parches de densidades TP35 y T3110 tras su corrección espacial y temporal. Hemos correlacionado los parches de ambas cartas que tienen el mismo valor o un valor muy próximo de densidad. Si la corrección es válida, debe haber una alta coincidencia en los promedios de los parches equivalentes o muy próximos en densidad. Se presentan los resultados en la tabla 2, contemplando procesado HDR y no HDR, y con una comparativa so- bre los datos antes de la corrección espacio temporal (ET). Para facilitar la comprensión, la diferencia se ha escalado a un rango tonal de 8 bits (de 0 a 255 valores).

A la vista de estos resultados, se puede concluir que la corrección espacio temporal (ET) mejora significativamente la correspondencia de valor de píxel promedio de las áreas que tienen la misma densidad ya estén sobre una misma imagen o en diferentes imágenes, por lo que es necesaria para poder conseguir una representación fiel a nivel densitométrico de los originales. Y esto es válido tanto para el procesado convencional como para el procesado HDR, si bien, el primero arroja mejores resultados, que son explicables por los procesados no lineales implicados para la obtención de una imagen HDR. Para apreciar el nivel de corrección ET, en la figura 6 mostramos la ecualización de las imágenes corregidas (ET) y las no corregidas HDR y no HDR. Usamos la ecualización para resaltar los cambios de intensidad en toda la superficie de la imagen.

En la figura 7 se muestra una comparativa de la calidad conseguible con el tipo de procesado que proponemos frente a un procesado convencional que no corrige inhomogeneidades ni problemas de rango dinámico. Se trata de una
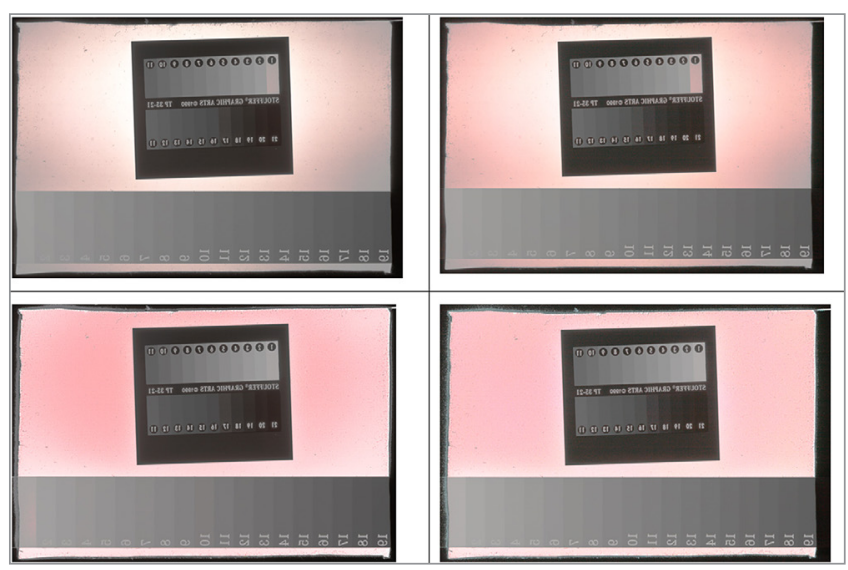

Figura 6. Comparativa de las imágenes ecualizadas de las dos cartas tonales: arriba, sin corrección ET (no HDR y HDR) y abajo las corregidas ET (no HDR y HDR). 
imagen negativa que presenta muy altas densidades, algo superiores a 2,4 od, y con muy poco contraste. Se ha positivado el negativo tratando además de acercar la imagen a lo que sería una copia positiva de época. El resultado de la corrección es excelente. La información extra del procesado HDR permite la aplicación de ajustes extremos de contraste en la versión positiva sin distorsión alguna. En la imagen sin corregir las relaciones tonales quedan desvirtuadas; por ejemplo, el suelo con las sombras de los caballos pierde su naturalidad.

\subsection{Generación de versiones positivas historicistas}

La realización de copias virtuales historicistas ha sido ya mencionada en trabajos anteriores (Martínez; Muñoz, 2002; Puglia; Reed; Rhodes, 2004) en los que se insiste en la necesidad de realizar un análisis histórico de la tecnología fotográfica de copia que pudo haber empleado el fotógrafo para generar versiones positivas de sus negativos. Se precisa indagar sobre los procedimientos fotográficos de copia más habituales en el entorno de trabajo del fotógrafo, pues se exige cierto conocimiento de las características tonales del procedimiento fotográfico de época elegido para la emulación, considerando atributos tales como el margen de densidades habitual, el contraste y los acabados de la superficie.

\section{Las copias virtuales historicistas requie- ren indagar sobre los procedimientos fo- tográficos de copia más habituales en el entorno de trabajo del fotógrafo}

La copia virtual en este planteamiento no es una mera inversión tonal y geométrica de la imagen negativa que porta el original negativo, sino que trata de aproximar, en la reproducción del fichero en una pantalla de monitor correctamente caracterizada y calibrada, la apariencia de una copia fotográfica de época contemplada en unas condiciones de visualización determinadas. Para poder conseguir este ideal se hace preciso incorporar la tecnología de gestión de color al procesado de la imagen positiva. Esta tecnología obliga a trabajar con las imágenes en un espacio de color conocido cuya gama de color y contraste sea suficiente como para contener el margen de densidades y color deseado para la copia virtual. Se ha elegido el espacio de color sRGB. Pese a la reducida gama de color y razón de contraste de este espacio, ha sido elegido porque permite representar sin problemas los márgenes de densidades de las imágenes del fondo y es el que suelen tomar por defecto la mayoría de aplicaciones de visualización de imágenes y navegadores web. Así aumenta la probabilidad de obtener visualizaciones correctas desde una amplia gama de aplicaciones web para todo tipo de usuarios. La reconstrucción historicista tiene sus límites, no se puede recrear todo el acabado del objeto copia positiva, pues nos inventaríamos elementos físicos que no tenemos en el negativo; lo máximo que se puede hacer es simular la apariencia de la imagen, pero sobre un soporte virtual que presenta exactamente los mismos elementos que el negativo.

Seguidamente se describen de forma sumaria los pasos seguidos para la creación de las copias historicistas:
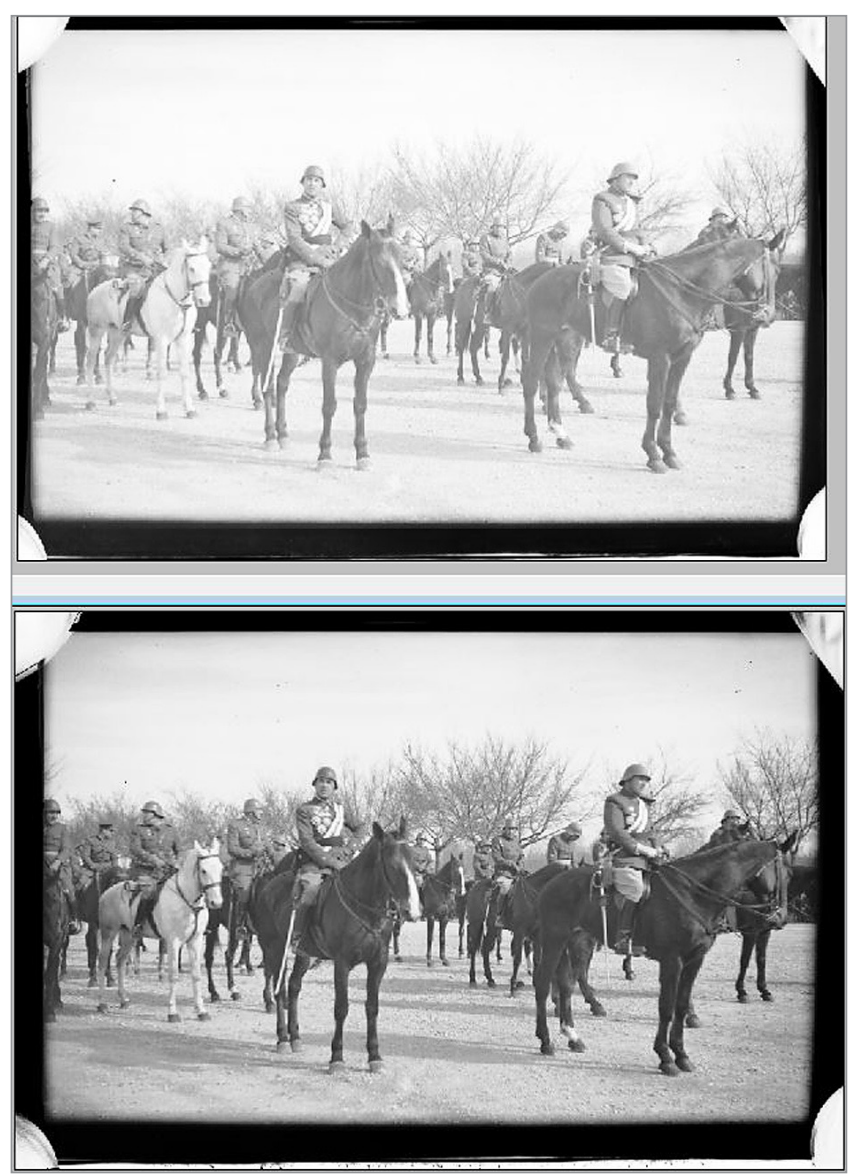

Figura 7. Imagen 1035 procesada en contraste para crear un positivado historicista. Arriba sin ningún tratamiento de corrección, abajo con corrección espacio temporal y procesado HDR

1) Elección de un procedimiento fotográfico al que emular. Se ha emulado una copia sobre soporte de papel baritado al gelatino bromuro de plata, procesada mediante revelado químico y sin virado alguno. La tonalidad de la imagen final es neutra, con una buena gradación tonal desde el negro al blanco.

2) Decisión de los márgenes de densidad y de la curva de contraste. Se ha abarcado un amplio margen de densidades en aquellos negativos con un alto contraste, amplificando el contraste aún más, pero sin perder la gama tonal de los medios tonos. Para ello se ha aplicado una tonal curva típica en $\mathrm{S}$, que oscurece los tonos más densos y aclara los más claros, llevando los tonos más cercanos al blanco casi hasta el límite del recorte, y manteniendo los tonos medios en su lugar.

3) Selección de las variables de salida. Se ha mantenido el espacio sRGB como espacio de salida, remuestreando los 16 bits a 8 bits. Se ha elegido el formato JPEG JFIF con compresión JPEG con pérdida con un nivel de calidad 11, manteniendo la misma resolución que el negativo para no perder detalle icónico.

4) Procesado, con las siguientes operaciones:

- enderezamiento de la imagen, corrigiendo el ángulo de inclinación con que ha sido capturada, en su caso;

- recorte de elementos accesorios de la captura ajenos al soporte; 
- ajuste de gamma 2,2;

- inversión tonal y geométrica del negativo original corregido ET y HDR;

- conversión a monocromo mediante la desaturación de la imagen;

- ajuste de contraste con las herramientas Histograma y Curvas;

- codificación con los valores de salida y creación del fichero JPEG.

En las figuras 8 y 9 se muestra el resultado de aplicar este procesado con dos copias virtuales historicistas obtenidas a partir de los negativos de la muestra con la que hemos trabajado ya corregidos de sus defectos de falta de uniformidad.

\section{Conclusiones}

Partiendo de la información de rendimiento del sistema de captura en parámetros tonales, hemos desarrollado y aplicado una serie de técnicas de corrección que han permitido corregir los problemas graves de inhomegeneidad espacial y temporal de las imágenes master, codificadas linealmente a 16 bits por canal tras un procesado raw básico. Si además usamos conjuntamente técnicas de procesado HDR, podemos conseguir en aquellas un amplio y correcto registro de los niveles de densidad de los originales, incluso empleando dispositivos de captura que no aportan los niveles de calidad requeridos.

Hemos demostrado asimismo que, con un conocimiento preciso de las características de los procedimientos fotográficos

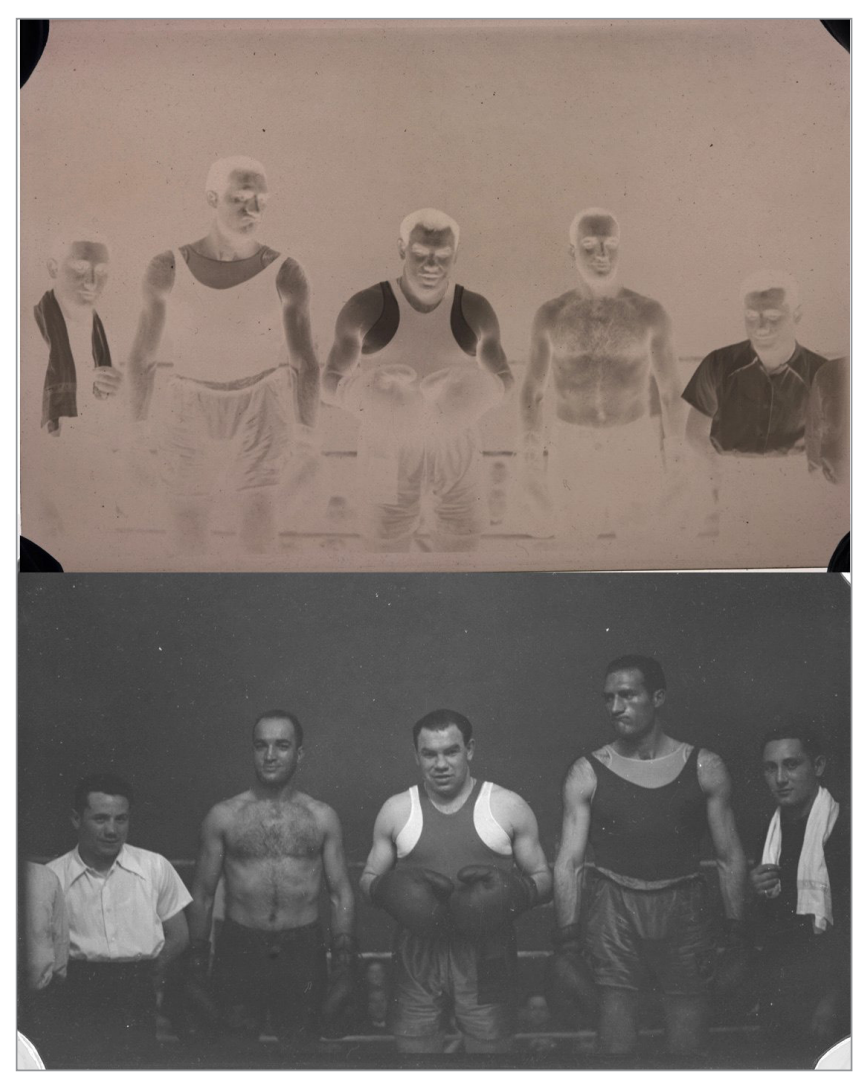

Figura 8. Copia virtual historicista de la imagen codificada como 1024 arriba la versión negativa codificada linealmente y sin ningún tipo de ajuste de corrección de copia habituales en la época de los negativos tratados, es posible implementar un sencillo procesado que, partiendo de imágenes digitales master de los negativos convenientemente corregidos y que presenten un registro correcto de todos los valores de densidad de los originales, genere versiones virtuales positivas que emulen lo que pudo haber sido una copia contemporánea a los negativos tratados.

\section{Notas}

1. Skogler fue la marca comercial del fotógrafo español Ángel Cortés Gracia, cuyo fondo de negativos se conserva en custodia en la Fototeca de la Diputación Provincial de Hues$c a$ y en manos de un coleccionista privado.

2. Todos los algoritmos ideados y descritos más abajo han sido implementados en Matlab y puestos a disposición de los lectores, junto a todos los ficheros de datos y una muestra de imágenes obtenidas y utilizadas para el estudio, en un sitio web público.

http://galan.uc3m.es/ jroble/skogler.zip

\section{Referencias}

Arjona, Maria-Montserrat; Novella, M. L.; De-Lasarte, Marta; Pujol, Jaume; Vilaseca, Meritxell (2004). “Caracterización espacial de dispositivos de adquisición de imágenes para su utilización en la medida de color". En: VII Congreso nacional de color 2004, pp. 173-174. ISBN: 8497690532

Brown, Michael S.; Seales, W. Brent (2001). “Document restoration using 3D shape: a general deskewing algorithm for

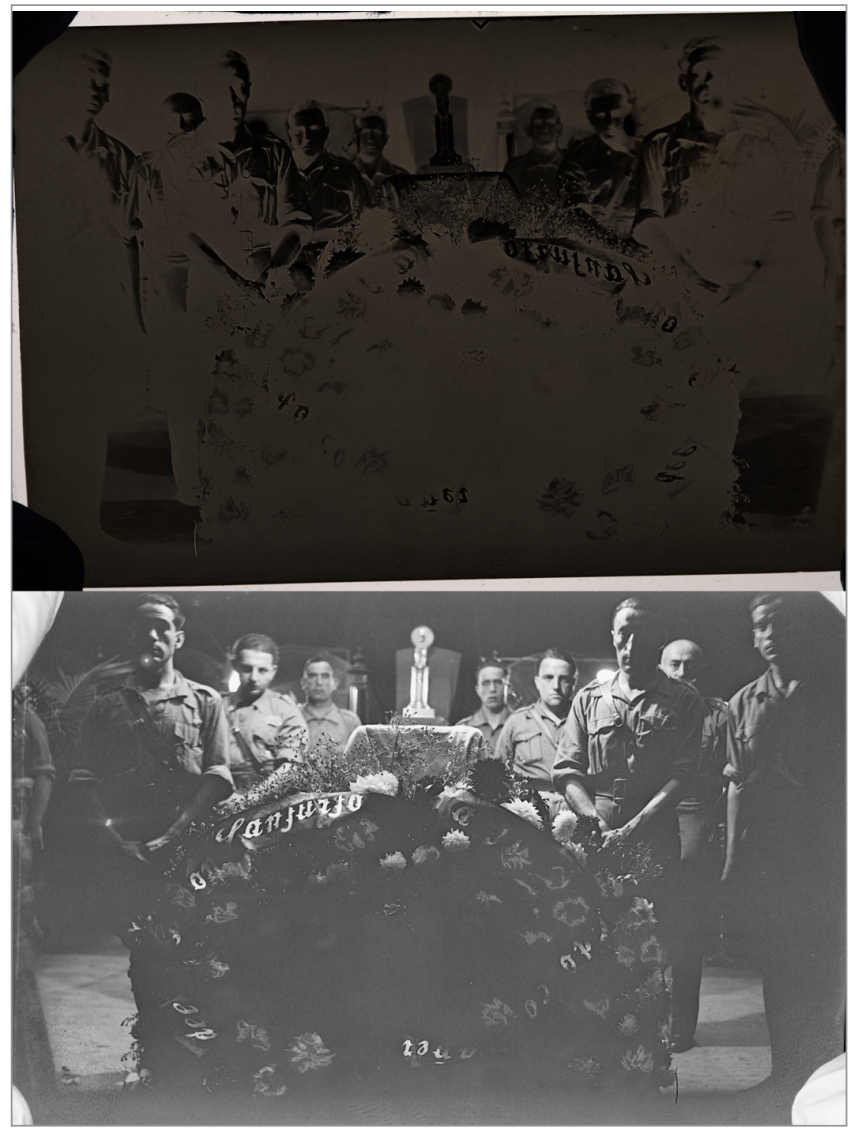

Figura 9. Copia virtual historicista de la imagen 1017, arriba la versión negativa codificada linealmente y sin ningún tipo de ajuste de corrección 
arbitrarily warped documents". En: Proceedings of the IEEE International conference on computer vision (ICCV '01), v. 2, pp. 367-374.

https://goo.gl/5WBNcP

https://doi.org/10.1109/ICCV.2001.937649

Brown, Michael S.; Seales, W. Brent (2004). "Image restoration of arbitrarily warped documents". IEEE Transactions on pattern analysis and machine intelligence, v. 26, n. 10, pp. 1295-1306.

https://www.comp.nus.edu.sg/ brown/pdf/pami-final-2004. pdf

\section{https://doi.org/10.1109/TPAMI.2004.87}

Cao, Huaigu; Ding, Xiaoqing; Liu, Changsong (2003). "A cylindrical surface model to rectify the bound document image". In: Procs of the $9^{\text {th }}$ IEEE Intl conf on computer vision (ICCV'03), v. 1, pp. 228-233.

https://goo.gl/VWEk15

https://doi.org/10.1109/ICCV.2003.1238346

Capell, Laura (2010). "Digitization as a preservation method for damaged acetate negatives: A case study". The American archivist, v. 73, n. 1, pp. 235-249.

https://doi.org/10.17723/aarc.73.1.x381802g137421h3

Fadgi - Still Image Working Group (2009). Technical guidelines for digitizing cultural heritage materials: Creation of raster image master files. For the following originals - manuscripts, books, graphic illustrations, artwork, maps, plans, photographs, aerial photographs, and objects and artifacts. Federal Agencies Digitization Initiative.

http://www.digitizationguidelines.gov/guidelines/FADGI_ Still_Image-Tech_Guidelines_2010-08-24.pdf

Fadgi - Still Image Working Group (2016). Technical guidelines for digitizing cultural heritage materials: Creation of raster image master files. September 2016. Federal Agencies Digitization Initiative.

https://goo.gl/8d217R

Frey, Franziska S.; Reilly, James M. (1999). Digital imaging for photographic collections: Foundations for technical standards. Rochester, NY: Image Permanence Institute.

Frey, Franziska S.; Reilly, James M. (2006). Digital imaging for photographic collections: Foundations for technical standards (2a ed.). Rochester, NY: Image Permanence Institute. https://www.imagepermanenceinstitute.org/webfm_ send/650

Hardie, Russell C.; Baxley, Frank O.; Brys, Brandon J.; Hytla, Patrick C. (2009). "Scene-based nonuniformity correction with reduced ghosting using a gated LMS algorithm". Optics express, n. 17, pp. 14918-14933.

https://goo.gl/GN9NPz

Hayat, Majeed M.; Torres, Sergio N.; Armstrong, Ernest; Cain, Stephen C.; Yasuda, Brian (1999). "Statistical algorithm for nonuniformity correction in focal-plane arrays". Applied optics, v. 38, n. 8, pp. 772-780. http://ece-research.unm.edu/hayat/ao.ps https://doi.org/10.1364/AO.38.000772

Huang, Jun; Yong, Ma; Fan, Fan; Xiaoguang, Mei; Liu, Zhe (2015). "A scene-based nonuniformity correction algorithm based on fuzzy logic". Optical review, v. 22, n. 4, pp. 614-622. https://doi.org/10.1007/s10043-015-0107-4

ISO (2017a). ISO/TR 19263-1:2017. Photography - Archiving systems. Part 1: Best practices for digital image capture of cultural heritage material. Geneva: ISO.

ISO (2017b). ISO/TR 19264-1:2017. Photography - Archiving systems - Image quality analysis - Part 1. Reflective originals. Geneva: ISO.

Landon, George V. (2015). "A pipeline for digital restoration of deteriorating photographic negatives". International journal on digital libraries, v. 16, n. 1, pp. 79-88.

https://doi.org/10.1007/s00799-014-0133-z

Landon, George V.; Clarke, Duncan; Seales, W. Brent (2009). "A new technique for the digitization and restoration of deteriorated photographic negatives". Eurasip journal on image and video processing, v. 2009:217016.

https://goo.gl/4o1BUV

https://doi.org/10.1155/2009/217016

Leong, F. Joel W-M; Brady, Michael; McGee, James O'D. (2003). "Correction of uneven illumination (vignetting) in digital microscopy images". Journal of clinical pathology, $\mathrm{v}$. 56, pp. 619-621.

https://www.ncbi.nlm.nih.gov/pmc/articles/PMC1770032

Likar, Bostjan; Maintz, J. B. Antoine; Viergever, Max A.; Pernuš, Franjo (2000). "Retrospective shading correction based on entropy minimization". Journal of microscopy, v. 197, n. 3, pp. 285-295.

https://doi.org/10.1046/j.1365-2818.2000.00669.x

Likar, Bostjan; Viergever, Max A.; Pernuš, Franjo (2001). "Retrospective correction of MR intensity inhomogeneity by information minimization". IEEE transactions on medical imaging, v. 20, pp. 1398-1410.

https://goo.gl/Af5X57

https://doi.org/10.1007/978-3-540-40899-4_38

Martínez, Celia; Muñoz, Jesús (2002). “Digitalización del patrimonio fotográfico e investigación: la metodología empleada para la reproducción digital de la colección de placas de vidrio de colodión húmedo, custodiada en el Museo Nacional de Ciencias Naturales - Consejo Superior de Investigaciones Científicas- (MNCN-CSIC)". En: Amador-Carretero, Pilar; Robledano-Arillo, Jesús; Ruiz-Franco, María-Rosario (eds.). Actas de las Primeras jornadas sobre imagen, cultura y tecnología, pp. 99-120.

https://e-archivo.uc3m.es/handle/10016/8933

Mitjà, Carles (2012). "Infraestructura tecnològica i procediments de treball per a la digitalització d'arxius fotogràfics".

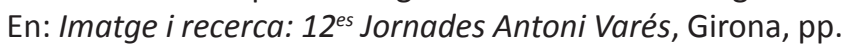
55-66.

https://goo.gl/9bsZtc

Nationaal Archief (2010). Digitisation of photographic materials. Guidelines. September.

http://www.nationaalarchief.nl/sites/default/files/docs/ guidelines_digitisation_photographic_materials.pdf

Puglia, Steven; Reed, Jeffrey; Rhodes, Erin (2004). Technical guidelines for digitizing archival materials for electronic 
access: Creation of production master files - raster images. For the following record types. Textual, graphic illustrations/ artwork/originals, maps, plans, oversized, photographs, aerial photographs, and objects/artifacts. NARA. U.S. National Archives and Records Administration.

https://www.archives.gov/files/preservation/technical/ guidelines.pdf

Puglia, Stephen; Rhodes, Erin (2007). “Digital imaging. How far have we come and what still needs to be done". RLG digiNews, v. 11, n. 1.

https://goo.gl/gy9C4W

Reilly, James M.; Frey, Franzisca S. (1996). Recommendations for the evaluation of digital images produced from photographic, microphotographic, and various paper formats. Report to the Library of Congress National Digital Library Project. Contract \# 96CLCSP7582.

http://lcweb2.loc.gov/ammem/lpireprt.pdf

Robledano-Arillo, Jesús (2011). “Mejora del rango dinámico en la digitalización de documentos desde una perspectiva patrimonial: evaluación de métodos de alto rango dinámico (HDR) basados en exposiciones múltiples". Revista española de documentación científica, v. 34, n. 3, pp. 357-384.

https://doi.org/10.3989/redc.2011.3.816

Robledano-Arillo, Jesús (2014). "Modelos de calidad en la digitalización de patrimonio fotográfico". En: Olivera-Zaldua, María; Salvador-Benítez, Antonia (eds.). Del artefacto mágico al píxel. Estudios de fotografía. XXIII Jornadas Fadoc. I Congreso intl de Documentación fotográfica. Madrid: Facultad de Ciencias de la Documentación de la UCM, pp. 367394. ISBN: 8469705313

https://goo.gl/1qq7yy

Robledano-Arillo, Jesús (2016). “25 años de conversión di- gital. Estado de la cuestión". En: 30 años de ciencia en la conservación de fotografías. Pamplona: CAAP, pp. 283-308. ISBN: 9788460846475

Robledano-Arillo, Jesús (2017). Captura digital de patrimonio cultural. Experiencia de trabajo con el fondo del archivo Espacio P. Granada: Ars Activus Ediciones. ISBN: 97884 94458828

https://e-archivo.uc3m.es/handle/10016/24283\#preview

Schoonees, Johann A.; Palmer G. Terry (2009). "Camera shading calibration using a spatially modulated field". En: $24^{\text {th }}$ Intl conf image and vision computing New Zealand (IVCNZ 2009).

http://kauri.auck.irl.cri.nz/ johanns/publications/shading_ calib_ivcnz09.pdf

Tomaževič, Dejan; Likar, Bostjan; Pernuš, Franjo (2002). "Comparative evaluation of retrospective shading correction methods". Journal of microscopy, v. 208, n. 3, pp. 212-223. https://goo.gl/RnLRiE

Van-Dormolen, Hans (2012). Metamorfoze preservation imaging guidelines. Image quality. Version 1.0, January 2012. The Hague, NL: National Library of the Netherlands (KB). https://goo.gl/WBr81H

Yu, Wonpil (2004). "Practical anti-vignetting methods for digital cameras". IEEE Transactions on consumer electronics, v. 50 , pp. $975-983$.

Zhang, Li; Zhang, Yu; Tan, Chew (2008). “An improved physically based method for geometric restoration of distorted document images". IEEE transactions on pattern analysis and machine intelligence, v. 30, n. 4, pp. 728-734. https://goo.gl/fST4Jm https://doi.org/10.1109/TPAMI.2007.70831
La Fundación Biblioteca Social es una institución sin ánimo de lucro que se constituyó en el año 2014. Tiene como objetivo contribuir a compensar los desequilibrios sociales apoyando proyectos que llevan a cabo las bibliotecas públicas, dirigidos a los sectores más vulnerables de la sociedad

\section{¿Colaboras?}

fundacionbibliotecasocial.org info@fundacionbibliotecasocial.org

3 @Biblio_Social

FundacionBibliotecasSocial

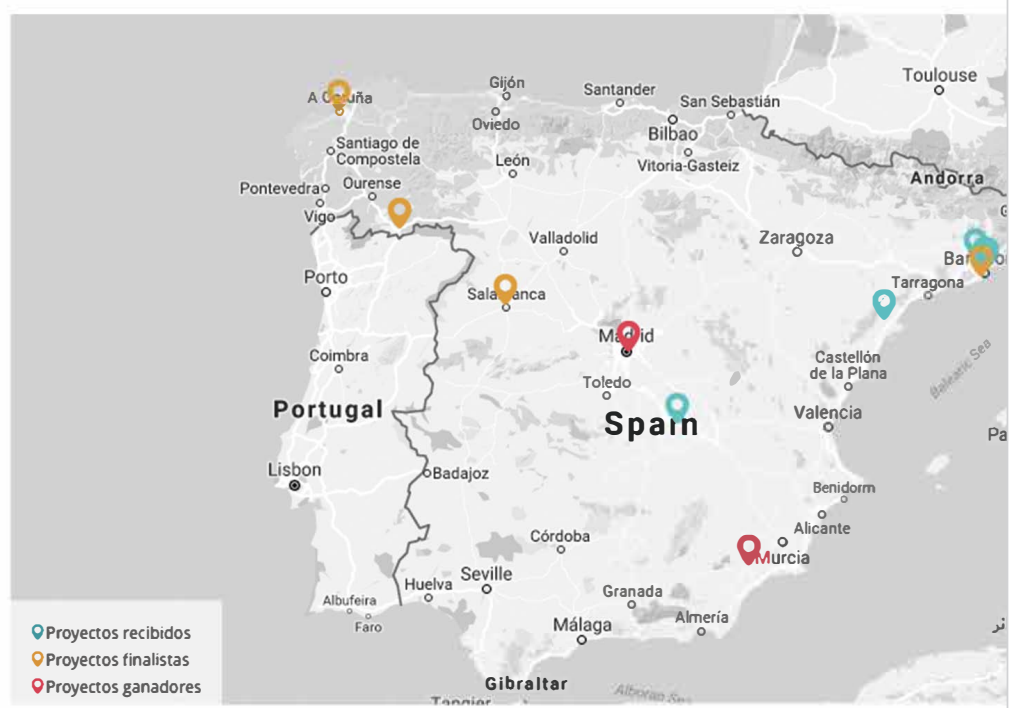

Mapa de proyectos de bibliotecas públicas para la inclusión social. 2016.

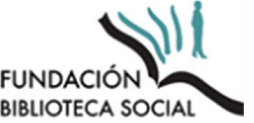

